\title{
Nota CienTífica \\ Diferencias morfométricas de dos especies del género Eatoniella en Isla Navarino, Reserva de Biosfera Cabo de Hornos, Chile
}

\author{
Morphometric differences in two species of the genus Eatoniella in \\ Navarino Island, Cape Horn Biosphere Reserve, Chile

\section{Sebastián Rosenfeld ${ }^{1,2}$, Cristian Aldea ${ }^{3}$, Jaime Ojeda ${ }^{1}$, Andrés Mansilla ${ }^{1,2}$ y Ricardo Rozzi ${ }^{2,4,5}$}

\begin{abstract}
${ }^{1}$ Laboratorio de Macroalgas Antárticas y Subantárticas, Universidad de Magallanes, Casilla 113-D, Punta Arenas, Chile. rosenfeld.sebastian@yahoo.com

${ }^{2}$ Instituto de Ecología y Biodiversidad (IEB), Santiago, Chile

${ }^{3}$ Programa GAIA-Antártica, Universidad de Magallanes, Casilla 113-D, Punta Arenas, Chile

${ }^{4}$ Universidad de Magallanes, Parque Etnobotánico Omora, Puerto Williams, Chile

${ }^{5}$ University of North Texas, Department of Philosophy and Religion Studies, Denton, Texas, USA

Abstract.- The external morphometrics of shell and radula morphology were performed in two species of micromollusks of the genus Eatoniella in Navarino Island, Chile. A total of 50 specimens of E. picea and 72 of E. nigra were analyzed. These species have similar morphology, but $E$. picea has a smaller diameter/length ratio $(0.53 \pm 0.02)$ than $E$. nigra $(0.61 \pm 0.04)$. In relation to the radula morphology, E. picea has wider middle cuspid teeth and small denticles in its border, meanwhile $\mathrm{E}$. nigra has pointed central cuspid teeth.
\end{abstract}

Key words: Micromollusks, morphology, shell, radula, Subantarctic

\section{INTRODUCCIÓN}

Dentro de los moluscos se consideran micromoluscos las especies en las cuales el individuo adulto no supera los $5 \mathrm{~mm}$ de longitud (Geiger et al. 2007). Aunque los moluscos son el grupo más diverso en el macrobentos litoral y sublitoral, para los que se dispone de abundantes datos taxonómicos comparativos (Valdovinos et al. 2003), la mayoría de los estudios sobre biodiversidad no incluyen información sobre micromoluscos (Albano et al. 2011). Esto se debe principalmente a su pequeño tamaño, que dificulta la identificación de las especies, contándose con escasa información ecológica o descriptiva. Probablemente existe un gran porcentaje de taxa que aún no han sido descritas o que aún conservan caracteres diagnósticos que no han sido estudiados (Geiger et al. 2007). Por ello, es relevante la información descriptiva y más si es útil para diferenciar sus especies, considerando la importancia que tienen los micromoluscos, desde el punto de vista del conocimiento de la biodiversidad y taxonomía (Sasaki 2008, Middelfart et al. 2016).

Entre los micromoluscos, uno de los grupos representativos son los gasterópodos del género Eatoniella Dall, 1876 (Gastropoda, Eatoniellidae). Este género representa a un grupo de diminutos gasterópodos risoiformes que se caracterizan externamente por presentar una concha oval cónica con una marcada inclinación en el labio externo y un opérculo que generalmente presenta un área de inserción opaca (Ponder \& Worsfold 1994).

En las costas del Pacífico Sur oriental, Atlántico Sur occidental, islas Subantárticas y la Península Antártica este grupo está representado por 24 especies (Ponder 1983, Worsfold et al. 1993, Ponder \& Worsfold 1994). Del total de especies conocidas, Ponder \& Worsfold (1994) reportaron 10 especies para la costa chilena, de las cuales 7 se registraron en la Provincia Magallánica y solamente 3 más al norte de la Provincia Magallánica: E. nigra d'Orbigny, 1840, E. glomerosa Ponder \& Worsfold, 1994 y E. mcleani Ponder \& Worsfold, 1994.

Dentro de la Provincia Magallánica las especies Eatoniella nigra y E. picea Ponder \& Worsfold, 1994 son representativas de ambientes intermareales (Ponder \& Worsfold 1994). E. picea fue descrita para el Estrecho de Magallanes y su distribución está restringida desde los $53^{\circ} \mathrm{S}$ hasta los $54^{\circ} \mathrm{S}$ (Ponder \& Worsfold 1994, Ojeda et al. 2014). Por su parte, E. nigra fue descrita bajo el nombre de Paludestrina nigra para el norte de Chile (d'Orbigny 1840). Posteriormente, Marincovich (1973) describió la especie E. latina, la cual constituía el primer registro de un representante de la familia 
Eatoniellidae para el Pacífico Oriental. Sin embargo, Ponder \& Worsfold (1994), al revisar las conchas de ambas especies, encontraron una morfología común, más baja, más ovoide y más delgada que las otras especies de color negro presentes en Sudamérica, por tanto, a partir de dicho trabajo, E. latina es considerada sinónimo de E. nigra. La especie está actualmente distribuida desde el norte de Chile hasta el Estrecho de Magallanes y por el lado Atlántico en la Isla de los Estados (Ponder \& Worsfold 1994).

A pesar que estas dos especies han sido señaladas como comunes en ambientes intermareales (Ponder \& Worsfold 1994), la información de estos microgasterópodos en la Provincia Magallánica aún es escasa. Por ejemplo, en estudios sobre la ecología o biodiversidad de las playas rocosas intermareales, los microgasterópodos no han sido reportados (e.g., Mutschke et al. 1998, Rios \& Mutschke 1999, Aldea \& Rosenfeld 2011, Rosenfeld et al. 2013). El único estudio que registró estas especies fue el análisis de patrones intermareales de Ojeda et al. (2014) en costas rocosas de Isla Navarino, canal Beagle. No obstante, es probable que estas especies sean más comunes en ensambles de moluscos intermareales subantárticos y su ecología aún no ha sido investigada. De esta manera, en el presente trabajo se realiza una comparación morfométrica de la relación diámetro/longitud (D/L) de las conchas de ambas especies con el fin de precisar un carácter externo que sirva de herramienta para facilitar el reconocimiento entre E. nigra y E. picea. Adicionalmente, se incorpora información comparativa sobre la morfología de sus rádulas.

\section{MATERIALES Y MÉTODOS}

El área de estudio se ubicó en Bahía Róbalo, Isla Navarino (545' S; 67³9'O), que se encuentra frente al brazo Noreste del Canal Beagle, formando parte de la Reserva de Biosfera Cabo de Hornos. El área se caracterizó por presentar piscinas intermareales, con bolones grandes y medios. Se realizó solo un muestreo en marzo 2010 durante baja marea. Se recolectaron 122 ejemplares vivos sobre algas coralinaceas y en frondas varadas de Lessonia flavicans. Del total de individuos recolectados, 72 correspondieron a Eatoniella nigra y 50 a E. picea. El muestreo se realizó siguiendo los protocolos entregados por Geiger et al. (2007), para colecta de micromoluscos.

Los especímenes recolectados fueron inmediatamente puestos en frascos plásticos y conservados en alcohol al 96\%. Posteriormente, los ejemplares fueron llevados al laboratorio para ser revisados e identificados, utilizando como referencia principal la revisión de Ponder \& Worsfold (1994). Para determinar la relación diámetro/longitud (D/L) se midió el ancho máximo, perpendicular a la altura de la concha, la cual corresponde a la longitud desde el ápice a la base. Para esto se midieron 30 ejemplares de cada especie. Las medidas fueron realizadas utilizando una lupa estereoscópica OLYMPUS $®$ CX31 adosada a una cámara y usando el software Micrometrics ${ }^{\circledR}$ SE. Adicionalmente, se utilizaron las medidas de diámetro y longitud de 10 individuos publicados en el trabajo de Ponder \& Worsfold (1994), para las especies E. nigra y E. picea, 6 individuos de E. nigra publicados por Marincovich (1973) para las costas de Iquique y 2 individuos de E. nigra publicados por Román (2005) para la localidad de Algarrobo.

Para la obtención de la rádula se siguió el método de Ponder \& Yoo (1978) modificado; éste consiste en la maceración del animal en hipoclorito de sodio $(\mathrm{NaClO})$ diluido y su posterior lavado en agua destilada. Las rádulas fueron montadas en portaobjetos, observadas y fotografiadas en un microscopio OLYMPUS® CX31 adosada a una cámara usando el software Micrometrics® SE.

Se utilizó la prueba de Kolgomorov-Smirnov con el fin de evaluar si los datos cumplen los supuestos de homecedasticidad y normalidad. Esta prueba es necesaria para poder analizar la varianza de los datos (Pedrosa et al. 2015). Además, se utilizó la prueba estadística t-Student para evaluar si existen diferencias significativas en la relación (D/L), con el software STATISTICA 7.0 (StatSoft 2004) ${ }^{1}$.

\section{RESULTADOS Y DISCUSIÓN}

Las características morfológicas de las conchas de Eatoniella picea y E. nigra (Fig. 1 a,b) corresponden a las descritas por Ponder \& Worsfold (1994). En relación a las rádulas observadas de las dos especies, la principal diferencia se encuentra en el diente central. E. picea tiene una cúspide media ancha y con pequeños dentículos en su extremo (Fig. 2a,c), mientras que $E$. nigra presenta una cúspide central aguda (Fig. 2 b,d).

\section{MORfometría de laS CONCHAS}

Los ejemplares de $E$. picea presentaron una longitud media de $2,07 \pm 0,08 \mathrm{~mm}$, un diámetro de $1,09 \pm 0,04 \mathrm{~mm}$ y su relación diámetro/longitud fue de $0,53 \pm 0,02$ (Tabla 1 ) mientras que los ejemplares de $E$. nigra presentaron una longitud media de $1,65 \pm 0,12 \mathrm{~mm}$, un diámetro de $1,00 \pm 0,05 \mathrm{~mm}$ y su relación diámetro/longitud fue de 0,61 $\pm 0,03$ (Tabla 1). La prueba de t-Student determinó que existen diferencias significativas en la relación diámetro/longitud $(\mathrm{t}=-11,25 ; P=0,001)$ entre ambas especies.

${ }^{1}$ StatSoft. 2004. Statistica for Windows. Computer program manual. StatSoft Inc, Tulsa, USA. 


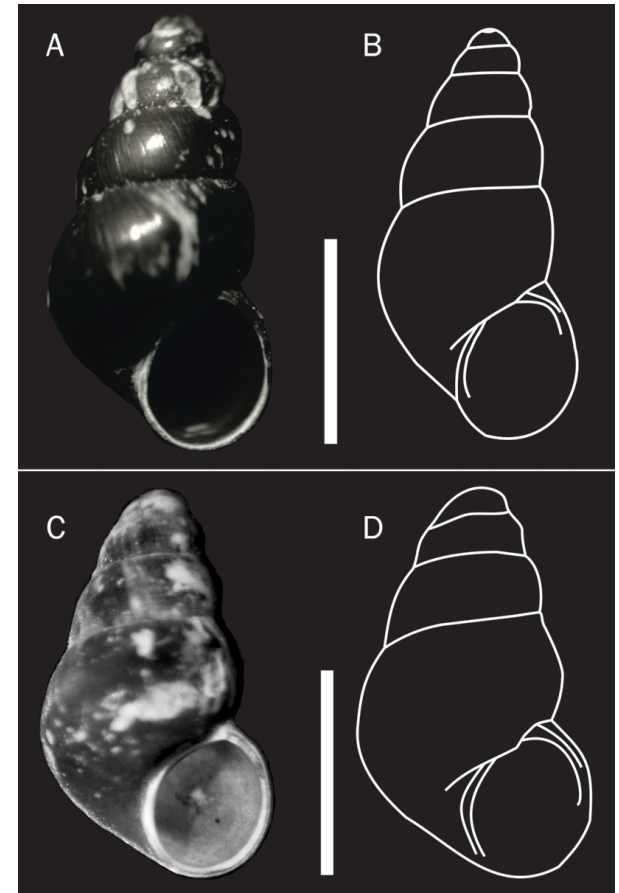

Figura 1. Vista general y dibujos de ejemplares de Eatoniella picea (AB) y E. nigra (C-D) de Bahía Róbalo, Isla Navarino Reserva de Biosfera Cabo de Hornos. Barras blancas $=1 \mathrm{~mm} /$ General view and drawings specimens of Eatoniella picea (A-B) and E. nigra (C-D) of Róbalo Bay, Navarino Island Cape Horn Biosphere Reserve. White bars $=1 \mathrm{~mm}$
Los ejemplares recolectados e identificados corresponden con los caracteres externos y forma de la rádula a la descrita por Ponder \& Worsfold (1994), y en E. nigra las medidas de longitud y diámetro de la concha son similares a las reportadas por Marincovich (1973) y Román (2005) (Tabla 1).

A pesar de que Ponder \& Worsfold (1994) realizaron estaciones de muestreo en Canal Beagle y Cabo de Hornos, no registraron especies de E. nigra ni tampoco de E. picea. Para la Provincia Magallánica el registro más al sur de E. nigra se reportó en una estación en Canal Cockburn $\left(54^{\circ} \mathrm{S}\right.$ y $\left.71^{\circ} \mathrm{O}\right)$ y para E. picea fue en el Estrecho de Magallanes. Por lo tanto, el trabajo realizado por Ojeda et al. (2014) y el registro entregado en este estudio corresponderían a los reportes de distribución más al sur de estas especies.

Por otra parte, mientras en E. picea el diámetro es apenas un poco más de la mitad de la longitud total, en E. nigra es alrededor del $60 \%$. Complementariamente, al calcular la relación D/L con las medidas de longitud y diámetro entregadas por Ponder \& Worsfold (1994), se pudo confirmar que E. nigra presenta una concha más ancha que E. picea (Tabla 1).

A pesar de la contribución realizada por Ponder \& Worsfold (1994) mediante la revisión morfológica y anatómica del género Eatoniella para la costa oriental del Pacífico Sur y parte del océano Atlántico, no existen estudios sobre la biodiversidad y ecología de estas especies en la Provincia Magallánica. Por ejemplo, Ponder \& Worsfold (1994) reporta E. picea, E. denticula, E. nigra y E. glomerosa como especies comunes,
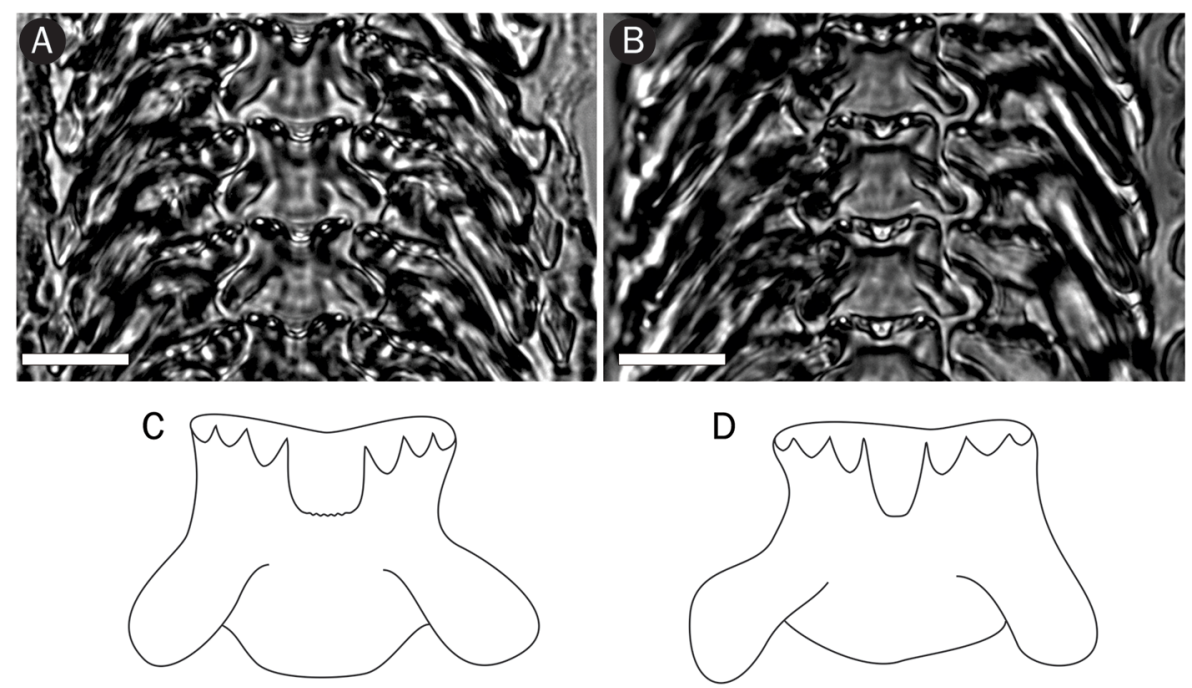

Figura 2. Vista general y dibujos de las rádulas de Eatoniella picea (A-C) y E. nigra (B-D) de Bahía Róbalo, Isla Navarino Reserva de Biosfera Cabo de Hornos. Barras blancas $=13 \mu \mathrm{m}$ / General view and drawings of the radulas of Eatoniella picea (A-C) and E. nigra (B-D) of Róbalo Bay, Navarino Island Cape Horn Biosphere Reserve. White bars= $13 \mu \mathrm{m}$ 
Tabla. 1. Comparación morfométrica de las especies E. picea y E. nigra colectadas en Bahía Róbalo, Isla Navarino ( $\mathrm{N}=30$ ). Se utilizaron las medidas morfométricas de las especies intermareales de Eatoniella que presentan una coloración gris o negra reportadas por: Ponder \& Worsfold (1994), Marincovich (1973) y Román (2005). L= longitud (mm), D= diámetro (mm) y D/ L= relación diámetro longitud / Morphometric comparison of the species E. picea and E. nigra collected in Róbalo Bay, Navarino Island $(\mathrm{N}=30)$. Morphometric measurements of black or grey intertidal Eatoniella species were used reported by: Ponder \& Worsfold (1994), Marincovich (1973) y Román (2005). L= length (mm), D= diameter (mm) and D/L= diameter length relationship

\begin{tabular}{lcccc}
\hline \multicolumn{1}{c}{ Especies } & Forma de las vueltas & $\mathrm{L}$ & $\mathrm{D}$ & $\mathrm{D} / \mathrm{L}$ \\
\hline Eatoniella picea & moderadamente convexas & $2,07 \pm 0,08$ & $1,09 \pm 0,04$ & $0,53 \pm 0,02$ \\
Eatoniella nigra $^{\text {Eatoniella picea }}{ }^{1}$ & suavemente convexas & $1,65 \pm 0,12$ & $1,00 \pm 0,05$ & $0,61 \pm 0,03$ \\
Eatoniella nigra $^{1}$ & & $2,28 \pm 0,11$ & $1,21 \pm 0,05$ & $0,53 \pm 0,02$ \\
Eatoniella nigra $^{2}$ & & $1,71 \pm 0,07$ & $1,02 \pm 0,04$ & $0,60 \pm 0,01$ \\
Eatoniella $^{3}$ nigra $^{3}$ & & $1,65 \pm 0,10$ & $1,04 \pm 0,07$ & $0,62 \pm 0,01$ \\
Eatoniella $^{2}$ ebenina $^{1}$ & & $1,58 \pm 0,01$ & $0,97 \pm 0,03$ & $0,61 \pm 0,02$ \\
\hline
\end{tabular}

${ }^{1}$ Ponder \& Worsfold (1994), ${ }^{2}$ Marincovich (1973), ${ }^{3}$ Román (2005)

las cuales, fueron muestreadas en el intermareal. Por el contrario, los trabajos intermareales de los últimos años (e.g., Mutschke et al. 1998, Rios \& Mutschke 1999, Aldea \& Rosenfeld 2011, Rosenfeld et al. 2013), no registran en sus listados ninguna especie del género Eatoniella. Otro factor que puede afectar la descripción de microgasterópodos es una incorrecta clasificación de los organismos comojuveniles de otras especies, ya que diversos autores han desarrollado técnicas especiales para la recolección y conservación de los micromoluscos (Geiger et al. 2007).

El hábitat donde se encontraron los ejemplares concuerda con lo descrito por Ponder \& Worlsfod (1994). Sin embargo, se encontraron microgasterópodos en una fronda de Lessonia flavicans Bory in Dumont d'Urville, 1825 varada en una piscina intermareal donde las rocas están cubiertas por algas coralinas. Al respecto, es importante destacar que este tipo de hábitats, favorecen la presencia de micromoluscos (Salas \& Hergueta 1986, Kelaher 2003). Estudios realizados en el alga calcárea Mesophyllum lichenoides (J. Ellis) Me Lemoine resaltan la presencia del pequeño tamaño de los ejemplares que habitan estas algas coralinaceas, apoyando la hipótesis del uso de estas formaciones como zonas de alta diversidad de micromoluscos (Salas \& Hergueta 1986). Esta interacción entre micromoluscos y algas coralináceas es notoria. En trabajos de selección de hábitat, e.g., Olabarria et al. (2002), realizaron un experimento en Australia con la especie Eatoniella atropurpurea (Frauenfeld, 1867), en el cual mencionan que debido a la capacidad que tienen las algas coralináceas de captar sedimento, radica la importancia para el desarrollo de estos microgastrópodos, esto porque se alimentan de las microalgas asociadas al sedimento, las que también sirven de refugio contra la depredación (Olabarria \&
Chapman 2001). Estudios de campo recientes han demostrado la importancia de las especies raras de microgastrópodos que habitan estas formaciones coralinaceas, ya que éstas tienen el potencial de contribuir de manera significativa a las funciones ecológicas, porque responden de la misma manera que las especies comunes (Matias et al. 2012). Por tanto, es importante generar un mayor conocimiento sobre la composición de los micromoluscos de la costa chilena, con el objetivo de abordar investigaciones ecológicas de campo (e.g., Olabarria et al. 2002, Chapman et al. 2008, Matias et al. 2012), que permitan tener una comprensión del funcionamiento de los ecosistemas marinos costeros (Matias et al. 2012).

Recientemente, ha habido un incremento en el conocimiento de la biodiversidad de micromoluscos (e.g., Sasaki 2008, Middlefart et al. 2016). Por ejemplo, en Australia de las 10 familias más diversas solamente 3 corresponden a macromoluscos, y las otras 7 corresponden a micromoluscos (Middlefart et al. 2016), mostrando que gran parte de su biodiversidad malacológica la componen micromoluscos.

Finalmente, se concluye que la diferencia morfológica en el ancho de la concha entre ambas especies es un punto comparativo para poder reconocer ambas especies. Las futuras investigaciones deben centrarse en las variaciones espaciotemporales de estas poblaciones en la zona intermareal de la Provincia Magallánica.

\section{Agradecimientos}

Al apoyo logístico brindado por la Universidad de Magallanes sede Puerto Williams representados por Dr. Francisca Massardo, MSc. Ximena Arango, MSc. Cristóbal Pizarro, 
Tamara Contador y Rosaria Civitelli. S. Rosenfeld agradece al IEB por la beca ICM, código P05-002. Finalmente se agradece a tres revisores anónimos del manuscrito por sus valiosos comentarios y aportaciones.

\section{LITERATURA CITADA}

Albano PG, B Sabelli \& P Bouchet. 2011. The challenge of small and rare species in marine biodiversity surveys: microgastropod diversity in a complex tropical coastal environmental. Biodiversity and Conservation 20: 3223-3237.

Aldea C \& S Rosenfeld. 2011. Moluscos intermareales de la Playa Buque Quemado (Estrecho de Magallanes, Chile). Revista de Biología Marina y Oceanografía 46: 115-124.

Chapman MG, AG Underwood \& D Blockley. 2008. Effects of method of deployment of artificial units of habitat on microgastropod colonization. Marine Ecology Progress Series 366: 49-57.

d'Orbigny A. 1840. Mollusques. Voyage dans l'Amérique Méridionale 5: 377-424.

Geiger D, B Marshall, W Ponder, T Sasaki \& A Warén. 2007. Techniques for collecting, handling, preparing, storing and examining small molluscan specimens. Molluscan Research 27(1): 1-50.

Kelaher B. 2003. Effects of frond length on diverse gastropod assemblages in coralline turf. Journal of the Marine Biological Association of the United Kingdom 83: 159-163.

Marincovich L. 1973. Intertidal molluscs from Iquique, Chile. Science Bulletin, Natural History Museum, Los Angeles County 16: 1-49.

Matias MG, MG Chapman, AJ Underwood \& NE O'Connor. 2012. Increasing density of rare species of intertidal gastropods: tests of competitive ability compared with common species. Marine Ecology Progress Series 453: 107-116.

Middelfart PU, LA Kirkendale \& NG Wilson. 2016. Australian Tropical marine micromolluscs: An overwhelming bias. Diversity 8(17): <doi:10.3390/d8030017>

Mutschke E, C Ríos \& A Montiel. 1998. Situación actual de la macrofauna presente en el intermareal de bloques y cantos de bahía Laredo, estrecho de Magallanes. Anales del Instituto de la Patagonia, Serie Ciencias Naturales, Chile 26: 5-9.

Ojeda J, S Rosenfeld, J Marambio, R Rozzi \& A Mansilla. 2014. Patrones estacionales y espaciales de la diversidad de moluscos intermareales de Bahía Róbalo, canal Beagle, Reserva de la Biosfera Cabo de Hornos, Chile. Revista de Biología Marina y Oceanografía 49(3): 493-509.
Olabarria C \& MG Chapman. 2001. Habitat-associated variability in survival and growth of three species of microgastropods. Journal of the Marine Biological Association of the United Kingdom 81: 961-966.

Olabarria C, AJ Underwood \& MG Chapman. 2002. Appropriate experimental design to evaluate preferences for microhabitat: an example of preferences by species of microgastropods. Oecologia 132: 159-166.

Pedrosa I, J Juarros-Basterretxea, A Robles-Fernández, J Basteiro \& E García-Cueto. 2015. Pruebas de bondad de ajuste en distribuciones simétricas, ¿qué estadístico utilizar? Universitas Psychologica 14(1): 245-254.

Ponder WF. 1983. Rissoaform gastropods from the Antarctic and sub-Antarctic: the Eatoniellidae, Rissoidae, Barleeidae, Cingulopsidae, Orbitestellidae and Rissoellidae (Mollusca: Gastropoda) of Signy Island, South Orkney Islands. British Antarctic Survey Scientific Reports 108: 1-96.

Ponder WF \& TM Worsfold. 1994. A review of the rissoiform gastropods of southwestern South America (Mollusca, Gastropoda). Contributions in Science, Natural History Museum of Los Angeles County 445: 1-63.

Ponder WF \& EK Yoo. 1977. A revision of the Eatoniellidae of Australia (Mollusca, Gastropoda, Littorinacea). Records of the Australian Museum 31: 606-658.

Ríos C \& E Mutschke. 1999. Community structure of intertidal boulder-cobble fields in the Strait of Magellan, Chile. Scientia Marina 63(1): 193-201.

Román R. 2005. Identificación, abundancia y distribución espacio-temporal de gastéropodos (juveniles y microgastéropodos) del submareal blando de la Rada el Algarrobo. Tesis de Biólogo Marino, Facultad de Ciencias del Mar y de Recursos Naturales, Universidad de Valparaíso, Viña de Mar, 235 pp.

Rosenfeld S, J Marambio \& C Aldea. 2013. Comparación de ensambles de moluscos en dos sustratos intermareales de la cuenca central del Estrecho de Magallanes. Amici Molluscarum 21(2): 7-18.

Sasaki T. 2008. Micromolluscs in Japan: taxonomic composition, habitats, and future topics. Zoosymposia 1: 147-232.

Valdovinos C, SA Navarrete \& PA Marquet. 2003. Mollusk species diversity in the Southeastern Pacific: why are there more species towards the pole? Ecography 26: 139-144.

Worsfold TM, G Avern \& WF Ponder. 1993. Shallow water rissoiform gastropods from Tristan da Cunha, South Atlantic Ocean, with records of species from Gough Island. Zoologica Scripta 22: 153-166. 